\title{
CARACTERIZAÇÃO DOS PADRÕES DE ESTRESSE HÍDRICO PARA A CULTURA DOARROZ (CICLO CURTO E MÉDIO) NO ESTADO DE GOIÁS E SUAS CONSEQÜÊNCIAS PARA O MELHORAMENTO GENÉTICO
}

\author{
Characterization of water stress patterns for rice (short and medium cycle) in Goiás State and \\ their consequences for the plant breeding program
}

\begin{abstract}
Alexandre Bryan Heinemann ${ }^{1}$
RESUMO

O estresse por deficiência hídrica é considerado como a maior restrição na produção e estabilidade da produtividade de culturas em muitas regiões do mundo. No Estado de Goiás predomina a produção de arroz de terras altas em sequeiro, sendo comum essa cultura sofrer períodos de deficiência hídrica ao longo do ciclo, que podem reduzir a produtividade. No processo de desenvolvimento de novas variedades, genótipos são selecionados em função de sua adaptabilidade em um determinado ambiente alvo. Assim, programas de melhoramento vegetal requerem informações sob a probabilidade de ocorrência e intensidade dos padrões de deficiência hídrica, associada as diferentes fases de desenvolvimento da cultura. Um modelo de simulação de culturas foi utilizado para determinar os padrões de deficiência hídrica no Estado de Goiás, considerando 12 locais e seis diferentes datas de semeadura para o arroz de ciclo curto e médio. Para ambos, as variedades estudadas, arroz ciclo curto e médio, quando semeados nas épocas favoráveis e sem impedimento ao desenvolvimento do sistema radicular, o estresse por deficiência hídrica não é uma restrição ao desenvolvimento da cultura no Estado de Goiás.
\end{abstract}

Termos para indexação: Modelos de simulação, cerrado, Oryza sativa, arroz de terras altas.

\begin{abstract}
Drought stress is a major constraint to crop production and yield stability in many regions of the world. In the state of Goiás, most of the rice crop system is rainfed. In a plant breeding program, new genotypes are selected as a result of their adaptability for a determined environment. Therefore, a plant breeding program needs information about drought frequency as well as its characteristics, intensity, and time during crop development. A crop simulation model was used to determine patterns of drought stress for 12 locations and six different planting dates for short and medium cycle rice. For upland rice, short and medium cycle, in favourable conditions (no soil impediment), drought stress is not a main constraint for the rice development in the state of Goiás.
\end{abstract}

Index terms: Crop model, Oryza sativa, savannah, upland rice.

(Recebido em 9 de janeiro de 2008 e aprovado em 26 de dezembro de 2008)

\section{INTRODUÇÃO}

A produtividade da cultura do arroz terras altas, na região Centro-Oeste, apresenta heterogeneidade temporal e espacial. Um dos principais fatores relacionados a produtividade que contribui para essa heterogeneidade é a disponibilidade de água no solo. Mesmo semeando o arroz na época das águas, existe a probabilidade de ocorrência de veranicos, períodos nos quais não há precipitação pluvial ou a precipitação pluvial é menor que a demanda, podendo expor a cultura a um período de estresse hídrico. Os efeitos negativos desse estresse podem ser intensificados se houver restrição ao crescimento radicular em razão da impedimentos físicos ou químicos, como alto teor de Al no subsolo.

Para melhorar o desempenho de genótipos de arroz de terras altas, em suas respectivas áreas de produção, em função de uma estratégia adaptativa a ser elaborada pelo programa de melhoramento, é necessário compreender as características da população de ambientes alvos para o programa de melhoramento e sua intravariabilidade potencial (TPE - "target population of environments"). TPE pode ser definida como um conjunto de ambientes (locais x manejo), incluindo sua variabilidade temporal e espacial, no qual os cultivares gerados por um programa de melhoramento devam apresentar um bom desempenho (Cooper et al., 1997). Por meio da caracterização das TPEs, um componente significante das interações genótipo $\mathrm{x}$ ambiente ( $\mathrm{G} \times \mathrm{A}$ ) para a produtividade pode ser explicado, permitindo a otimização de ambientes e metodologias de seleção (Chapman et al., 2000a,b).

Para uma estratégia de melhoramento relacionada com a tolerância à seca ter uma maior probabilidade de êxito, é essencial que os melhoristas conheçam a

1Embrapa Arroz e Feijão - Rod. GO-462, km 12 - 75375-000 - Santo Antônio de Goiás, GO - alexbh@cnpaf.embrapa.br 
probabilidade de ocorrência dos diferentes tipos de deficiência hídrica, bem como suas características como intensidade e duração e época de ocorrência em função da fase fenológica da cultura. Trata-se, portanto, da caracterização ambiental, levando-se em consideração, não somente os aspectos climáticos básicos como precipitação pluvial, mas também, os efeitos da interação G x A. Conhecendo-se as frequências dos padrões de deficiência hídrica de diferentes locais, as produtividades dos genótipos dos ensaios regionais podem ser ponderadas, de acordo com a sua representatividade na TPE. Esse tipo de estratégia de seleção é conhecida como seleção indireta (Cooper \& Podlich, 1999).

Estudos recentes para a caracterização de TPEs em função dos padrões de deficiência hídrica têm utilizado modelos de simulação de crescimento de culturas. Para a cultura do sorgo, Muchow et al. (1996) mostraram que modelos de simulação do crescimento de culturas foram mais efetivos na qualificação e quantificação dos diferentes padrões de deficiência hídrica que simples modelos de balanço hídrico do solo. Chapman et al. (2000b) utilizaram o modelo APSIM para criar uma tipologia regional dos padrões de estresse hídrico baseado em informações de solo e séries de dados climáticos, em Queensland, Austrália.

Neste estudo, objetivou-se caracterizar as variações geográficas e temporais dos padrões de estresse por deficiência hídrica para a cultura do arroz, ciclo curto e médio, e os seus respectivos impactos no melhoramento genético do arroz de terras altas no Estado de Goiás.

\section{MATERIAL E MÉTODOS}

\section{Caracterização da área de estudo}

Neste trabalho definiu-se como área de estudo o Estado de Goiás, cujo clima é definido como quente, semiúmido, com uma precipitação pluvial anual em torno de $1600 \mathrm{~mm}$, concentrada em sete a oito meses (Nimer, 1989). Doze diferentes locais (Aragarças, Ceres, Sto. Antônio de Goiás, Goiânia, Iporá, Itaberaí, Itumbiara, Planaltina, Porangatu, Quirinópolis, Vianópolis e Vicentinópolis) foram selecionados para estabelecer a base de dados climáticos e, consequentemente, a TPE. As informações da localização geográfica, altitude, número de anos da serie de informações meteorológicas utilizados dos respectivos locais estão ilustradas na Tabela 1. As variáveis diárias meteorológicas; temperatura máxima e mínima do ar, precipitação pluvial, umidade relativa máxima e mínima do ar, velocidade do vento e radiação global foram obtidas da rede de estações do SECTEC/SIMEHGO (Secretaria do Estado de Ciência e Tecnologia - Goiás). Esse conjunto de dados meteorológicos foram organizados em uma base de dados e submetidos a um processo de verificação de sua qualidade, como também, o preenchimento de dados perdidos ou nulos de acordo com a metodologia descrita em Heinemann et al. (2007). Na área de estudo, o solo mais frequente, representando $46 \%$ da área total, é o Latossolo (Embrapa, 1999), caracterizado pela boa permeabilidade e aeração, porém com baixa fertilidade natural e baixa capacidade de troca cationnica, frequentemente responsáveis por deficiências nutricionais nas culturas. Sua capacidade média de retenção de água é de aproximadamente $100 \mathrm{~mm} \mathrm{~m}^{-1}$ (Schaffert et al., 2000; Balbino et al., 2001). Nesse tipo de solo, há limitação para o desenvolvimento do sistema radicular de algumas culturas, principalmente, decorrente do elevado nível de Alumínio (Al) tóxico nas camadas subsuperficiais do perfil do solo. Assim, dois cenários, foram criados; a) solo profundo (solo com capacidade de água disponível de $80 \mathrm{~mm}$ para uma profundidade efetiva de $0,8 \mathrm{~m}$ ) e, b) solo raso (solo com capacidade de água disponível de $40 \mathrm{~mm}$ para uma profundidade efetiva de $0,4 \mathrm{~m}$ ), que representa a condição em que há impedimento físico para o desenvolvimento do sistema radicular (geralmente devido ao efeito da toxidez de $\mathrm{Al}$ ).

\section{Modelo de simulação de crescimento e desenvolvimento da cultura}

O modelo de crescimento RICE06, implementado na plataforma de modelos ECOTROP (Sultan et al., 2005; Kouressy et al., 2008), foi parametrizado para duas variedades de arroz, uma de ciclo curto e outra de ciclo médio, em função dos dados experimentais obtidos das variedades Guarani e Caiapó, respectivamente. Somente detalhes relevantes à versão do modelo utilizado serão descritos nesse estudo. Esse modelo é determinístico, com passos diários de cálculo. Foi desenvolvido baseado no antigo modelo SARRA, utilizado para análise de risco de safra para as culturas de sorgo e milheto na África Oriental (Affholder, 1997).

\section{Dinâmica da água no solo}

O modelo utilizado simula o balanço hídrico diário no solo. O solo é dividido em duas camadas, sendo a primeira camada, geralmente de $10 \mathrm{~cm}$, utilizada basicamente para o cálculo da evaporação, e a segunda (profundidade definida pelo usuário) representa a profundidade máxima que o sistema radicular poderá alcançar. A quantidade de água disponível no solo (CAD) é baseada na diferença entre a "capacidade de campo" e o "ponto de murcha permanente" e provém de análise laboratorial, sendo considerada nesse estudo $100 \mathrm{~mm}$ $\mathrm{m}^{-1}$. A profundidade da zona efetiva radicular depende das características genótipicas e do estádio fenológico da cultura e é limitada de acordo com a cultura, solo e profundidade de umedecimento do solo. A extração da água do solo consiste, basicamente, em dois componentes, evaporação pela superfície do solo e extração pelas raízes, via transpiração. 
Tabela 1 - Municípios e suas respectivas; coordenadas geográficas (latitude e longitude, em graus), altitude (m) e número de anos utilizados no estudo (n).

\begin{tabular}{lcccr}
\hline \multicolumn{1}{c}{ Local } & Latitude & Longitude & Altitude $(\mathrm{m})$ & $\mathrm{n}$ \\
\hline Aragarças & $-16,00$ & $-52,00$ & 310 & 6 \\
Sto A. de Goiás & $-16,50$ & $-49,30$ & 741 & 22 \\
Ceres & $-15,33$ & $-49,60$ & 739 & 6 \\
Goiânia & $-16,73$ & $-49,25$ & 749 & 6 \\
Iporá & $-16,41$ & $-51,11$ & 688 & 6 \\
Itaberai & $-16,01$ & $-49,78$ & 1001 & 6 \\
Itumbiara & $-18,40$ & $-49,18$ & 449 & 6 \\
Planaltina & $-16,08$ & $-47,70$ & 1007 & 6 \\
Porangatu & $-13,30$ & $-41,11$ & 391 & 6 \\
Quirinópolis & $-18,43$ & $-50,40$ & 633 & 6 \\
Vianópolis & $-16,80$ & $-48,48$ & 1110 & 6 \\
Vicentinópolis & $-17,70$ & $-49,78$ & 648 & 6 \\
\hline
\end{tabular}

Intensidade do estresse por deficiência hídrica na planta

A deficiência hídrica da planta é avaliada por meio da fração da água disponível do solo na zona das raízes, removida via transpiração. O modelo calcula separadamente a transpiração da evaporação. A variável FTSW, que é a relação entre a lâmina de água transpirada e a lâmina de água disponível na zona efetiva radicular, normalizada para a escala 0 a 1, é calculada diariamente (Sinclair \& Ludlow, 1986). Essa variável atua como um fator de redução no cálculo da transpiração e assimilação de carbono, utilizando o fator de depleção $p$ (Allen et al., 1998). A intensidade do estresse por deficiência hídrica na planta é representado pela variável CSTR (transpiração real/ transpiração potencial, variando de 0 a 1 ).

\section{Características das simulações}

As simulações foram efetuadas levando-se em consideração as datas de semeadura do arroz no Estado de Goiás. As datas de semeadura para o arroz de ciclo curto e médio foram de 1 de novembro a 31 de dezembro e de 15 de outubro a 31 de dezembro, respectivamente, com intervalos de 15 dias para ambos os ciclos. $\mathrm{O}$ arroz de ciclo médio possui um período maior de oportunidade de semeadura em razão da maior duração do período vegetativo. Especificamente para os locais Planaltina e Vicentinópolis, as datas de semeadura foram restritas ao mês de novembro, em razão do menor período chuvoso. Decorrente do fato de as datas de semeadura terem sido fixadas a priori no modelo, independente da ocorrência de chuva, uma restrição foi imposta, permitindo que a emergência ocorresse somente se a umidade na primeira camada do solo fosse maior ou igual a $70 \%$ da capacidade de campo, nos dez dias subsequentes à semeadura. $\mathrm{O}$ início das simulações se deu em julho, para permitir um estabelecimento mais realístico do perfil de água do solo, baseado nas ocorrências de precipitação antes das datas de semeadura.

\section{Tipologia dos padrões de estresse hídrico}

Para o estabelecimento dos padrões de deficiência hídrica para o arroz ciclo curto e médio, foi estabelecida uma matriz constituída de local, data de semeadura e ano (representando as linhas) e a média dos valores do CSTR (intensidade do estresse por deficiência hídrica na planta), definido como a razão entre a transpiração real e transpiração potencial, para cada período de 100 GD (Graus Dias, temperatura base de $10^{\circ} \mathrm{C}$ ) do desenvolvimento da cultura (representando as colunas). A essa matriz aplicouse o método de classificação denominado "cluster". Esse método é hierárquico e de aglomeração, baseado na distância euclidiana como medida de dissimilaridade e soma dos quadrados como critério de fusão (Ward, 1963). Para cada TPE, ciclo curto e longo, as tipologias das deficiências hídricas foram classificadas em três principais grupos baseados na similaridade do CSTR em função do desenvolvimento fenológico simulado da cultura. Para evitar bias nas análises dos padrões de deficiência hídrica, decorrente do fato de o modelo superestimar a evaporação no início do desenvolvimento da cultura, considerou-se somente para análise o período do ciclo da cultura entre 400 GD (meio do período vegetativo) e o final do enchimento dos grãos, para o ciclo curto, e $500 \mathrm{GD}$ ao final 
do período de enchimento de grãos, para o ciclo médio. Metodologia de classificação similar foi utilizada e descrita por Muchow et al. (1996), Chapman et al. (2000a) e Heinemann et al. (2010).

As variáveis de saída do modelo utilizadas foram: CSTR (transpiração real/transpiração potencial), produtividade potencial simulada (PP - sem deficiência hídrica), produtividade real simulada (PR). A partir das variáveis PP e PR, calculou-se o impacto da deficiência hídrica na produtividade, dado pela seguinte equação (1):

$$
I R S H=100\left(1-\frac{P R}{P P}\right)
$$

em que, IRSH se refere ao impacto da deficiência hídrica na produtividade, em $\%, P R$ a produtividade real simulada, em $\mathrm{kg} \mathrm{ha}^{-1}$, e $P P$ a produtividade potencial simulada, em $\mathrm{kg} \mathrm{ha}^{-1}$.

O programa estatístico utilizado neste estudo foi o R v.2.4.0 (R Development Core Team, 2005), utilizando-se o pacote "Cluster".

\section{RESULTADOS E DISCUSSÃO}

\section{Caracterização dos padrões de deficiência hídrica - arroz ciclo curto}

Para o arroz de ciclo curto foram determinados três diferentes padrões de deficiência hídrica em função da análise de cluster realizada (Figura 1a). Esses três padrões de deficiência hídrica foram denominados L (sem deficiência hídrica), M (deficiência hídrica mediana na fase reprodutiva) e $\mathrm{T}$ (deficiência hídrica na fase terminal). $\mathrm{O}$ padrão de deficiência hídrica $\mathrm{T}$ foi o mais severo, ocasionando uma redução na produtividade de $53 \%$ e $62 \%$, para os cenários solos profundo e raso, respectivamente (Tabela 2). Esse padrão inicia-se na fase reprodutiva (500 GD) e tem a sua maior intensidade no final dessa fase (800 GD), uma semana antes da data de florescimento, estendendo-se, também, na fase de enchimento de grãos. A partir do florescimento, esse padrão de deficiência hídrica torna-se mais intenso para o solo raso (Figura 1a). Entretanto, esse padrão apresentou a menor frequência de ocorrência, $16 \%$ e $29 \%$, para os solos profundo e raso, respectivamente.

O padrão de deficiência hídrica $\mathrm{M}$ ocasionou uma redução na produtividade de $18 \%$ e $32 \%$, para os solos profundo e raso, respectivamente (Tabela 2). Esse padrão inicia-se no começo da fase reprodutiva (500 GD) e tem a sua maior intensidade também nessa fase, a 600 GD. A partir dessa fase, sua intensidade diminui, principalmente para o cenário solo profundo. Para o cenário solo raso, esse padrão é o predominante na TPE, apresentando a maior frequência de ocorrência $(48 \%)$. O padrão de deficiência hídrica L, para ambos os cenários, apresentou a menor redução na produtividade, $6 \%$ e $10 \%$ para os solos profundo e raso, respectivamente. Esse padrão é o mais frequente para o solo profundo, representando $46 \%$ dos casos de ocorrência, é o menos frequente para o solo raso, $23 \%$ dos casos de ocorrência.

A data de semeadura que apresenta a menor probabilidade de ocorrência do padrão de deficiência hídrica T é 15 de novembro, para ambos os cenários. A partir dessa data, há uma tendência no aumento da ocorrência desse padrão de deficiência hídrica. As falhas na emergência decorrentes da falta de umidade no solo, foram observadas somente no início do período de semeadura (Figura 2a).

\section{Caracterização dos padrões de deficiência hídrica - arroz ciclo médio}

Para o arroz de ciclo médio, também foram determinados três diferentes padrões de deficiência hídrica em função da análise de cluster realizada (Figura 1b). Esses três padrões de deficiência hídrica foram denominados $\mathrm{L}$ (sem deficiência hídrica), V (deficiência hídrica na fase vegetativa) e MT (deficiência hídrica na fase reprodutiva e terminal). O padrão de deficiência hídrica MT é o mais severo, ocasionando uma redução na produtividade de $51 \%$ e $66 \%$, para os solos profundo e raso, respectivamente (Tabela 2). Esse padrão tem início no meio da fase vegetativa (600 GD) e apresenta a sua maior intensidade nas fases reprodutiva e enchimento de grãos. Esse padrão apresentou a menor frequência de ocorrência para o cenário solo profundo, $12 \%$, e a segunda maior para o cenário solo raso, $24 \%$ (Tabela 2 ).

$\mathrm{O}$ padrão de deficiência hídrica $\mathrm{V}$ ocasionou uma redução na produtividade de $10 \%$ e $27 \%$, para os solos profundo e raso, respectivamente (Tabela 2 ). Esse padrão inicia-se no meio da fase vegetativa e apresenta a sua maior intensidade, também, nessa fase, a 600 GD. A partir dessa fase, sua intensidade diminui, principalmente para o cenário solo profundo. Para o solo raso, esse padrão apresenta a mesma frequência de ocorrência que o padrão L, 44\%. Para o solo raso, esse é o padrão de deficiência hídrica predominante na TPE, apresentando a maior frequência de ocorrência (59\%).

O padrão de deficiência hídrica L, para ambos os cenários, apresentou a menor redução na produtividade, $3 \%$ e $10 \%$ para os solos profundo e raso, respectivamente. Para solo raso, é o menos frequente, representando somente $17 \%$ dos casos de ocorrência. 

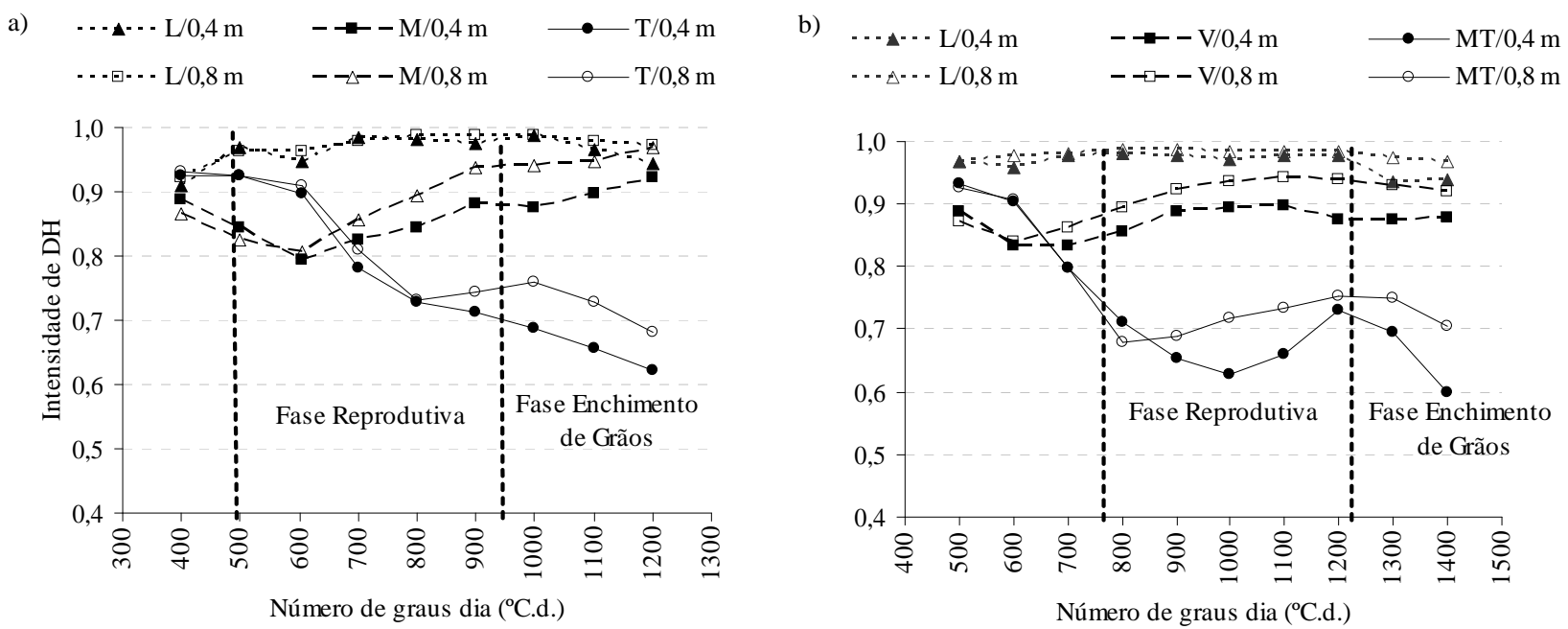

Figura 1 - Padrões de deficiência hídrica (DH) e suas intensidades em função das fases de desenvolvimento da cultura de arroz ciclo curto (a) e médio (b), sendo L - sem deficiência hídrica; $M$ - deficiência hídrica mediana; $\mathrm{T}$ - deficiência hídrica terminal; V - deficiência hídrica na fase vegetativa e MT - deficiência hídrica na fase reprodutiva e terminal.

Tabela 2 - Produtividade potencial (sem deficiência hídrica), produtividade real (com deficiência hídrica), impacto da deficiência hídrica na produtividade (IRSH) e padrões de deficiência hídrica (sem deficiência hídrica - L, deficiência hídrica mediana - M, deficiência hídrica terminal - $\mathrm{T}$, deficiência hídrica na fase vegetativa - $\mathrm{V}$ e deficiência hídrica na fase reprodutiva e terminal - MT) e seus respectivos desvios padrões (dp) para as TPEs de arroz.

\begin{tabular}{|c|c|c|c|c|c|c|c|c|c|}
\hline \multirow{3}{*}{ Solo } & \multirow{3}{*}{ Variável } & \multicolumn{4}{|c|}{ Arroz (ciclo curto) } & \multicolumn{4}{|c|}{ Arroz (ciclo médio) } \\
\hline & & \multicolumn{3}{|c|}{$\begin{array}{c}\text { Padrões de deficiência } \\
\text { hídrica }\end{array}$} & \multirow[t]{2}{*}{ Média } & \multicolumn{3}{|c|}{$\begin{array}{c}\text { Padrões de deficiência } \\
\text { hídrica }\end{array}$} & \multirow[t]{2}{*}{ Média } \\
\hline & & $\mathrm{L}$ & $\mathrm{M}$ & $\mathrm{T}$ & & $\mathrm{L}$ & $\mathrm{V}$ & MT & \\
\hline \multirow{6}{*}{$\begin{array}{l}\text { Profundo } \\
0,8 \mathrm{~m}\end{array}$} & Produtividade potencial $\left(\mathrm{kg} \mathrm{ha}^{-1}\right)$ & 3937 & 4120 & 4217 & 4051 & 2950 & 2885 & 2860 & 2911 \\
\hline & $\mathrm{dp}\left(\mathrm{kg} \mathrm{ha}^{-1}\right)$ & 718 & 622 & 542 & & 483 & 485 & 498 & \\
\hline & Produtividade real $\left(\mathrm{kg} \mathrm{ha}^{-1}\right)$ & 3718 & 3378 & 1982 & 3311 & 2853 & 2597 & 1402 & 2566 \\
\hline & $\mathrm{dp}\left(\mathrm{kg} \mathrm{ha}^{-1}\right)$ & 785 & 834 & 942 & & 535 & 720 & 914 & \\
\hline & IRSH (\%) & 6 & 18 & 53 & 18 & 3 & 10 & 51 & 12 \\
\hline & Freqüência da deficiência hídrica (\%) & 46 & 38 & 16 & & 44 & 44 & 12 & \\
\hline \multirow{6}{*}{$\begin{array}{l}\text { Raso } \\
0,4 \mathrm{~m}\end{array}$} & Produtividade potencial $\left(\mathrm{kg} \mathrm{ha}^{-1}\right)$ & 3927 & 4027 & 4270 & 4074 & 2976 & 2873 & 2956 & 2935 \\
\hline & $\mathrm{dp}\left(\mathrm{kg} \mathrm{ha}^{-1}\right)$ & 677 & 668 & 590 & & 407 & 493 & 514 & \\
\hline & Produtividade real $\left(\mathrm{kg} \mathrm{ha}^{-1}\right)$ & 3516 & 2742 & 1634 & 2599 & 2683 & 2086 & 1018 & 1929 \\
\hline & $\mathrm{dp}\left(\mathrm{kg} \mathrm{ha}^{-1}\right)$ & 728 & 869 & 944 & & 560 & 773 & 737 & \\
\hline & IRSH (\%) & 10 & 32 & 62 & 36 & 10 & 27 & 66 & 33 \\
\hline & Frequiência da deficiência hídrica (\%) & 23 & 48 & 29 & & 17 & 59 & 24 & \\
\hline
\end{tabular}

O padrão de estresse L, para ambos os solos, apresentou o menor impacto no rendimento, $3 \%$ e $10 \%$ para os solos profundo e raso, respectivamente. Para solo raso, é o menos frequente, representando somente $17 \%$ dos casos de ocorrência.
As datas de semeadura entre $1 / 11$ a $15 / 12$ apresentaram as menores probabilidades de ocorrência do padrão de deficiência MT, para ambos os cenários. O início da época de semeadura apresentou a maior probabilidade de ocorrência de falha na emergência (Figura 2b). 

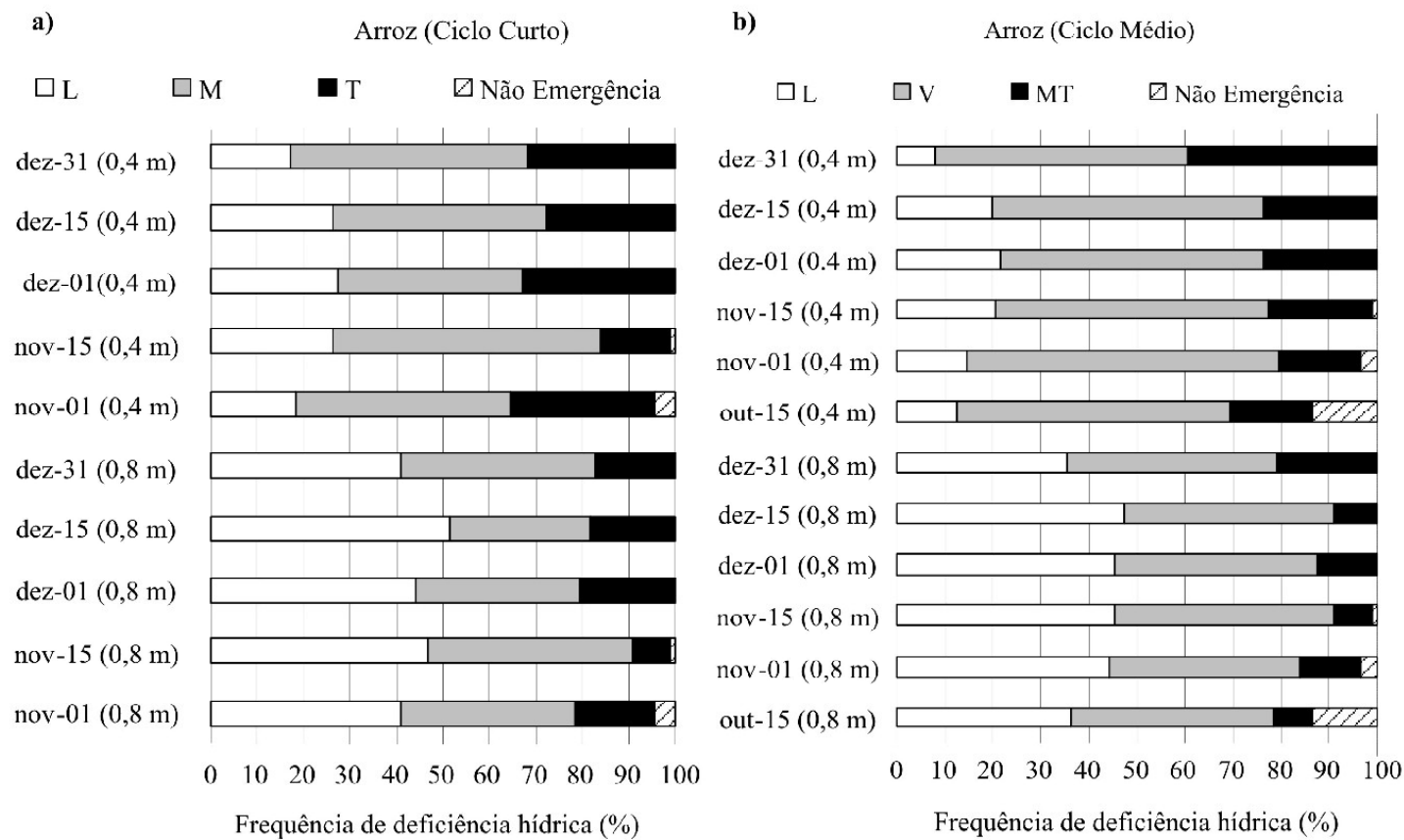

Figura 2 - Frequência dos diferentes padrões de deficiência hídrica em função das datas de semeadura e cenários (solo com 0,4 e 0,8 m de profundidade efetiva), para a cultura do arroz (a) ciclo curto e (b) ciclo médio, sendo (L) sem deficiência hídrica; (M) deficiência hídrica mediana; (T) deficiência hídrica terminal; (V) deficiência hídrica na fase vegetativa e (MT) deficiência hídrica na fase reprodutiva e terminal.

\section{Impacto da deficiência hídrica no programa de melhoramento genético de arroz terras altas}

Fukai \& Cooper (2001), em função da severidade e predição, identificaram três diferentes mecanismos possíveis em condições de estresse por deficiência hídrica: produtividade potencial, escape da época crítica (precocidade) e tolerância a seca. No caso de estresse por deficiência hídrica moderada, a produtividade potencial é o determinante mais importante na produtividade de uma determinada variedade. Pantuwan et al. (2002), também observaram que em condições de estresse moderado (variação na produtividade em torno de $20 \%$ ), a produtividade real é mais afetado pela produtividade potencial do que pelos efeitos da deficiência hídrica. Sob deficiência hídrica severa (redução na produtividade em torno de 50\%), a correlação entre produtividade em condição sem deficiência hídrica e com deficiência hídrica não é mais significativa (Ceccarelli \& Grando, 1991). Portanto, nessas condições, os genótipos são incapazes de expressar seu desenvolvimento potencial (Pantuwan et al., 2002). Assim, prevalecem mecanismos de escape (variedades precoces) e ou implantação de um programa de melhoramento com estratégia voltada a tolerância a seca.
Considerando-se os resultados obtidos nesse estudo, a redução na produtividade para a TPE do arroz indicou reduções no IRSH, em razão da deficiência hídrica, em média, menores que $50 \%$ para ambos os cenários, solo profundo e raso (Tabela 2). Para o cenário solo profundo, a soma da frequência de ocorrência dos padrões de deficiência hídrica L (para ambos os ciclos, curto e médio), $\mathrm{M}$ (ciclo curto) e V (ciclo médio) representam $84 \%$ e $88 \%$. Os padrões de estresse por deficiência hídrica que ocasionaram reduções na produtividade maiores que $50 \%$ ( $\mathrm{T}$, para o ciclo curto e MT para o ciclo médio) apresentaram uma frequência menor que $20 \%$ (16\% e $12 \%$, respectivamente para o ciclo curto e médio). Embora qualquer critério para a avaliação dos efeitos da severidade do estresse por deficiência hídrica na produtividade seja arbitrário, os resultados de simulação obtidos nesse estudo suportam a estratégia de melhoramento adotada pelo CNPAF, baseada, principalmente, no rendimento potencial, especialmente em solos que não apresentam impedimento físico ou químico. Além disso, o desempenho da produtividade de cultivares de terras altas (Pinheiro et al., 2006), a partir da década de 90, suporta essa afirmação. Consequentemente, para o cenário solo profundo, deficiência hídrica não é a principal restrição para ser 
abordado pelo programa de melhoramento de arroz de terras altas no Estado de Goiás, especialmente considerando-se as datas "ótimas" de semeadura. Para o cenário solo raso, que tem como objetivo representar propriedades com um menor nível tecnológico, consequentemente, apresentam solos com possíveis impedimentos químicos ou físicos, há uma maior ocorrência de padrões de deficiência hídrica mais drásticos. Nesse cenário, caracterizado por padrões de deficiência hídrica $\mathrm{M}$ e $\mathrm{V}$, com probabilidades de ocorrência de $48 \%$ para o ciclo curto e $59 \%$ para o médio, apresentam um impacto na produtividade (IRSH) de $36 \%$ e $33 \%$, para os ciclos curto e médio. Nessas condições, seria interessante a implementação da seleção indireta (Cooper \& Podlich, 1999), no qual, a produtividade de genótipos, nos diferentes ensaios de VCU, são ponderados em função do tipo padrão de estresse ocorrido durante o período de ensaio. Essa metodologia poderá auxiliar na seleção de linhagens mais adaptadas à condição de deficiência hídrica mais frequente encontrado na TPE.

\section{CONCLUSÕES}

A metodologia utilizada nesse estudo pode ser aplicada para a determinação de padrões de estresses em um determinado ambiente-alvo. Entretanto, o sucesso dessa metodologia é dependente da disponibilidade e qualidade da base de dados meteorológicos.

Para o arroz de ciclo curto e médio cultivados em solos sem impedimento ao desenvolvimento do sistema radicular, a deficiência hídrica não é uma restrição ao desenvolvimento das culturas no Estado de Goiás.

Para regiões que apresentam impedimento ao desenvolvimento do sistema radicular, é interessante aplicar a seleção indireta, considerando os tipos de padrões de deficiência hídrica que ocorrem nos ensaios regionais de seleção.

\section{REFERÊNCIAS BIBLIOGRÁFICAS}

AFFHOLDER, F. Empirically modelling the interaction between intensification and climatic risk in semiarid regions. Field Crops Research, Amsterdam, v.52, p.7993, 1997.

ALLEN, R.G.; PEREIRA, L.S.; RAES, D.; SMITH, M. Crop evapotranspiration-guidelines for computing crop water requirements. Rome: FAO, 1998. (FAO. Irrigation and Drainage Paper, 56).

BALBINO, L.C.; BRUAND, A.; BROSSARD, M.; GUIMARÃES, M. de F. Comportement de la phase argileuse lors de la dessiccation dans des Ferralsols microagrégés du Brésil: rôle de la microstructure et de la matière organique. Comptes Rendus de L' Academie des Sciences. Serie I: Sciences de La Terre et des Planètes, Montrouge, n.332, p.673-680, 2001.

CECCARELLI, S.; GRANDO, S. Environment of selection and type of germplasm in barley breeding for lowyielding conditions. Euphytica, Wageningen, v.57, p.207219, 1991.

CHAPMAN, S.; COOPER, M.; HAMMER, G.L.; BUTLER, D.G. Genotype by environment interactions affecting grain sorghum: II., frequencies of different seasonal patterns of drought stress are related to location effects on hybrid yields. Australian Journal Agricultural Science, Sydney, v.51, p.209-221, 2000a.

CHAPMAN, S.; HAMMER, G.L.; BUTLER, D.G.; COOPER, M. Genotype by environment interactions affecting grain sorghum: III., temporal sequences and spatial patterns in the target population of environments. Australian Journal Agricultural Science, Sydney, v.51, p.223-233, 2000b.

COOPER, M.; PODLICH, D.W. Genotype x environment interactions, selection response and heterosis. In: COORS, J.G.; PANDEY, S. (Eds.). The genetics and exploitation of heterosis in crops. Madison: ASA, 1999. p.81-92.

COOPER, M.; STUCKER, R.E.; DELACY, I.H.; HARCH, B.D. Wheat breeding nurseries, target environments, and indirect selection for grain yield. Crop Science, Madison, v.37, p.1168-1176, 1997.

EMPRESA BRASILEIRA DE PESQUISA

AGROPECUÁRIA. Centro Nacional de Pesquisa de Solos. Sistema brasileiro de classificação de solos. Brasília, 1999. 412p.

FUKAI, S.; COOPER, M. Development of drought resistant cultivars for rainfed lowland rice experience from Northeast Thailand and surrounding areas. In: INTERNATIONAL CONFERENCE ON THE IMPACT OF AGRICULTURAL RESEARCH FOR

DEVELOPMENT IN SOUTHEAST ASIA, 2000,

Phnom Penh. Proceedings... Phnom Penh, 2001. p.185-194. 
HEINEMANN, A.B.; SILVA, S.C. da; LOPES JÚNIOR, S.; AMORIM, A. de O.; ANDRADE, C. de L.T. de; BASTOS, E.A.; PAZ, R.L. da. Características climáticas dos municípios de Santo Antônio de Goiás (GO), Porangatu (GO), Janaúba (MG), Sete Lagoas (MG), Parnaíba (PI) e Teresina (PI), Brasil. Santo Antônio de Goiás: Embrapa Arroz e Feijão, 2007. 36p. (Embrapa Arroz e Feijão. Documentos, 214).

HEINEMANN, A. B. ; ANDRADE, C. de L. T. de; GOMIDE, R. L. ; AMORIM, A. de O. ; PAZ, R. L. da. Padrões de deficiência hídrica para a cultura de milho (safra normal e safrinha) no estado de Goiás e suas consequencias para o melhoramento genético. Ciência e Agrotecnologia, Lavras, v. 33, n.4, p.1026-1033, jul./ago., 2009.

KOURESSY, M.; DINGHUN, M.; VAKSMAN, M.; HEINEMANN, A.B. Adaptation to diverse semi-arid environments of sorghum genotypes having different plant type and sensitivity to photoperiod. Agricultural and Forest Meteorology, Amsterdam, v.148, p.357-371, 2008.

MUCHOW, R.C.; COOPER, M.; HAMMER, G.L. Characterizing environmental challenge using models. In: COOPER, M.; HAMMER, G.L. (Eds.). Plant adaptation and crop improvement. Wallingford: CABI, 1996. p.349-364.

NIMER, E. Climatologia do Brasil. Rio de Janeiro: IBGE, 1989. 421p.

PANTUWAN, G.; FUKAI, S.; COOPER, M.; RAJATASEREEKULC, S.; O'TOOLED, J.C. Yield response of rice (Oryza sativa L.) genotypes to drought under rainfed lowlands 2. Selection of drought resistant genotypes. Field Crop Research, Amsterdam, v.73, p.169-180, 2002.

PINHEIRO, B. da S.; CASTRO, E. da M. de; GUIMARÃES, C.M. Sustainability and profitability of aerobic rice production in Brazil. Field Crops Research, Amsterdam, v.97, p.34-42, 2006.

R DEVELOPMENT CORE TEAM. R: a language and environment for statistical computing. Vienna: R Foundation for Statistical Computing, 2005.

SCHAFFERT, R.E.; ALVES, V.M.C.; PARENTONI, S.N.; RAGHTHAMA, K.G. Genetic control of phosphorus uptake and utilization efficiency in maize and sorghum under marginal soil conditions. In: RIBAUT, J.M.; POLAND, D. (Eds.). Molecular approaches for the genetic improvement of cereals for stable production in water-limited environments: a strategic planning workshop held at CIMMYT. Mexico: CIMMYT, 2000.

SINCLAIR, T.R.; LUDLOW, M.M. Influence if soil water supply on the plant water balance of four tropical grain legumes. Australian Journal of Plant Physiology, Victoria, v.13, p.329-341, 1986.

SULTAN, B.; BARON, C.; DINGKUHN, M.; JANICOT, S. Agricultural impacts of large-scale variability of the West African monsoon. Agricultural and Forest Meteorology, Amsterdam, v.128, p.93-110, 2005.

WARD, J.H. Hierarchical grouping to optimize an objective function. Journal of the American Statistical Association, Washington, v.58, p.236-244, 1963. 